\title{
Human Papilloma Virus: Biomolecular Aspect
}

\section{Patiyus Agustiansyah ${ }^{1,2^{\star}}$, Rizal Sanif ${ }^{1}$, Siti Nurmaini $^{3}$, Irfannuddin ${ }^{4}$, Legiran $^{5}$}

${ }^{1}$ Department of Obstetric and Gynecology, Division Oncology of Gynecology, Faculty of Medicine, Universitas Sriwijaya / General Hospital Dr Moh. Hoesin, Palembang, Indonesia

${ }^{2}$ Doctoral Student, Biomedical Program, Faculty of Medicine, Universitas Sriwijaya, Palembang, Indonesia

${ }^{3}$ Intelligent System Research Group, Universitas Sriwijaya, Palembang, Indonesia

${ }^{4}$ Department of Physiology, Faculty of Medicine, Universitas Sriwijaya, Palembang, Indonesia

${ }^{5}$ Department of Anatomy, Faculty of Medicine, Universitas Sriwijaya, Palembang, Indonesia

\author{
A R T I C L E I N F O \\ Keywords: \\ HPV \\ Cervical Cancer \\ Biomolecular
}

*Corresponding author:

Patiyus Agustiansyah

E-mail address:

fatiyusagustiansyah@gmail.com

All authors have reviewed and approved

the final version of the manuscript.

\begin{abstract}
A B S T R A C T
HPV is a sexually transmitted virus, and high-risk HPV DNA was found in $99.7 \%$ of cervical cancer specimens. Within 12 to 24 months of exposure to the virus, $90 \%$ of HPV infections disappear or become inactive. However, infection with highrisk strains of HPV persist which then increases the risk of progression to cervical cancer. The detection of precancerous lesions consists of various methods, including pap smear (conventional or liquid-base cytology / LBC), visual inspection of acetic acid (IVA), visual inspection of lugoliodine (VILI), and HPV DNA test (genotyping / hybrid capture).
\end{abstract}

https://doi.org/10.32539/bsm.v5i8.327

\section{Introduction}

HPV is a sexually transmitted virus, and high-risk HPV DNA was found in $99.7 \%$ of cervical cancer specimens. ${ }^{1-6}$ Within 12 to 24 months of exposure to the virus, $90 \%$ of HPV infections disappeared or became inactive. However, infection with high-risk strains of HPV persist which then increases the risk of progression to cervical cancer. The detection of precancerous lesions consists of various methods, including pap smear (conventional or liquid-base cytology / LBC), visual inspection of acetic acid (IVA), visual inspection of lugoliodine (VILI), and HPV DNA testing (genotyping / hybrid capture. ${ }^{7-9}$
The pathogenesis of HPV infection involves the overexpression of viral oncoproteins which can inhibit various cellular proteins and influence biological processes including cell proliferation, cell cycle, and apoptosis. ${ }^{11}$ Changes in viral and host cells that cause cervical carcinogenesis provide deep insight into the nature of the disease as well as inspire therapeutic development. specific molecular targets. ${ }^{12-13}$

Infection by high-risk human papillomavirus (HPV) and integration of the HPV genome into host chromosomes of cervical epithelial cells are key early events in the development of neoplastic cervical lesions. Viral oncoproteins, especially E6 and E7, are 
responsible for the initial changes in epithelial cells. Viral proteins deactivate the two main tumor suppressor proteins, $\mathrm{p} 53$, and retinoblastoma $(\mathrm{pRb})$. Inactivation of this host protein interferes with the DNA repair mechanism and apoptosis, leading to rapid cell proliferation. Several genes involved in DNA repair, cell proliferation, growth factor activity, angiogenesis, and mitogenesis genes are highly expressed in cervical intraepithelial neoplasia (CIN) and cancer. This genomic instability prompts HPV-infected cells to develop into invasive carcinoma. ${ }^{14-17}$

HPV is classified in the Papillomaviridae family with the family divided into 2 subfamilies with more than 50 genera. Of the 50 genera, only 5 can infect humans, and the genus Alphapapillomavirus is the most common in patients. Apart from genus, HPV is divided into 3 based on the level of malignancy, namely mild, potentially high risk, and high risk (Figure 1). The causes of cervical cancer are HPV 16 and $18 .{ }^{18}$ While in Indonesia, based on Mudiyarso et al's research, the prevalence of HPV infection in cervical cancer patients is mostly in types $16,18,45$, and $52 .{ }^{19}$.

\section{Pathogenesis of cervical cancer}

The natural history of HPV infection and the occurrence of cervical cancer begins with the presence of cervical precancerous lesions revealed cytologically (low grade and high grade) and histopathologically (Cervical Intraepithelial Neoplasia or CIN1, CIN2, CIN3) ${ }^{20}$ (Figure 2). The process of tumorigenic transformation from viral entry to invasive carcinoma takes 10-20 years and thus provides a window period for treating the disease in its early stages. The initial infection is mainly latent, subclinical, and opportunistic. Genital HPV infection is very common in young, sexually active women. Furthermore, sexual contact with an individual infected with HPV increases the likelihood of contracting the virus. Some additional risk factors include use of oral contraceptives, a history of sexually transmitted infections, smoking, and immunosuppression. Various factors depending on their role in the development of carcinogenic disease can be classified into exposure-related, host-related susceptibility, factors affecting the tumor microenvironment, and somatic / epigenetic. Among the factors that strongly influence HPV transmission and pathogenicity are the prevalence of different HPV strains in certain populations, duration of infection, patterns of sexual contact and pathogenic behavior of the infecting virus. ${ }^{21}$

The HPV virus is a double stranded virus or dsDNA without a sheath and enters the human body through microabrasion. HPV then binds to the primary Syndecan-1 receptor (Heparan Sulfate Proteglycans (HPGs) isotype) then performs endocytosis. This bond causes the viral DNA to come out of the vesicles and binds to the microfilaments and helps transport in the cytoplasm and nucleus cells.Then, the viral capsid codes in the form of L1 and L2 are formed, making it possible to carry out exocytosis or other target cell infections.

The human papilloma virus infects only epithelial cells and relies on the epithelial cell differentiation pathway to complete its life cycle. The human papilloma virus infects cells in the basal layer of the epithelium, possibly through microabrasion on the epithelial surface. Internalization of the infection takes several hours, after which the viral DNA is released from the capsid and transported into the nucleus as free genetic material or extrachromosomal episodes. Initial gene expression was tightly controlled in basal epithelial cells with substantial amplification of viral DNA. Replication (figure 3) occurs only in suprabasal, distinct cells that are destined to mature and mature, and therefore do not naturally express the replicative machinery that the virus relies on to survive. To avoid this problem, the human papillomavirus encodes two proteins - E6 and E7 - that together promote cell proliferation, prolong cell cycle progression, and prevent apoptosis. Cells become permissive for viral replication and hundreds or even thousands of human papillomavirus genomes are generated in a single cell. The capsid proteins L1 and L2 are expressed in the most superficial layer of the epithelium, where the viral assembly occurs, and finally, new infectious virus particles (virions) are released from the epithelial 
surface (Figure 4). The papillomavirus life cycle takes 2-3 weeks, the time it takes for cervical cells to migrate from the basal to the most superficial layers of the epithelium, mature, age, and die. ${ }^{22}$

The structure of the HPV virus is divided into 3 , namely the Upstream Regulatory Region (URR) by $15 \%$, the early region $45 \%$, and the late region $40 \%$. The invasion begins with the binding of cellular transcription factors, replication, and transcription. The first target cells are basal cells in the cervical epithelium and their activity is affected as the maturation of the host cells increases. This means that the replication process is slow and less productive when the cells occupy the basal part of the epithelium. However, as the host epithelium matures, HPV genome replication increases and the initiation of a cascade encoding E6 and E7 as well as L1 and L2. The end result is that new immortal cells and virions are formed to infect other cells that have not been infected.

HPV as an initiating factor of cervical cancer which causes cervical cell disorders. The integration of viral DNA with the body's cell genome is the beginning of the process that leads to transformation. The HPV genome is circular and $8 \mathrm{~kb}$ long, has 8 open reading frames (ORFs) and is divided into early (E) and late (L) genes. Gen E synthesizes 6 E proteins, namely E1, E2, E4, E5, E6 and E7, which are widely involved in the replication process of viruses and oncogens, while the $\mathrm{L}$ gene synthesizes $2 \mathrm{~L}$ proteins namely L1 and L2 which are associated with capsid formation.

The life cycle of the papillomavirus is different from all other viral families: infection requires the availability of viable reproductive epidermal or mucosal epithelial cells (basal layer cells). In these cells, viral gene expression is largely suppressed, although limited expression of certain early viral genes (such as E5, E6 and E7) results in increased proliferation of infected cells and their lateral expansion. After entering the suprabasal layer, the expression of viral genes slowly begins; the circular viral genome is then replicated and structural proteins are formed. In the upper layer of the epidermis or mucosa, complete viral particles collect and are released.
Three genes have proliferation stimulating activity, namely E5, E6 and E7. E5 is important in the early course of infection, as it stimulates cell growth by forming a complex with epidermal growth factor receptors, the platelet-derived growth-factor-beta receptor and factor-1 receptor. that stimulates the colony (the colony-stimulating factor-1 receptor). Recently, E5 has also been shown to prevent apoptosis after DNA damage. However, when an HPV-infected lesion develops cervical cancer, the episomal viral DNA often becomes integrated into the DNA of the host cell, and a large part of the genome, generally including the E5 code sequence, is deleted. Thus, E5 is not mandatory in the event of late HPV-mediated carcinogenesis. HPV protein E5 also plays a role in supporting persistent HPV infection. The E5 protein causes downregulation of NK cell receptors. The downregulation of receptors results in NK cells unable to attach to their receptors so that their activity in eliminating cancer cells will decrease. The decrease in NK cell activity causes several cytokines that can trigger the adaptive immune response to not secrete. E6 protein, which consists of 150 amino acids, binds to the cellular protein E6-associated protein (E6-AP) to form the Ubiquitin Ligase enzyme complex. This enzyme complex causes the degradation of p53. Degradation of p53 causes normal activity of p53 such as stopping the cell cycle after the G1 phase, apoptosis, and no DNA repair. In addition, E6 protein also plays a role in inducing the c-myc protein to trigger the activity of the telomerase enzyme. As a result, cells will become immortal because the telomeres do not shorten.

Protein E7 consists of 100 amino acids to form a complex with RB protein which is hypophosphorylated causing interference with the pRB complex and cellular transcription factor $\mathrm{E} 2 \mathrm{~F}-1$. As a result, the transcription factor E2F-1 is free and detached from the DNA strand, resulting in transcription of the genes required to enter the $\mathrm{S}$ phase of the cell cycle and inhibits apoptosis of the host cell. The host cell becomes immortal and continues to divide uncontrollably. In normal circumstances, changes in cells will trigger an immune response so that the 
abnormalities at this stage can be overcome and the abnormal cells are in apoptosis. Naturally, virusinfected cells will release type 1 interferon (IFN) such as IFN- $\alpha$ and IFN- $\beta$. Interferons inhibit viral replication in host cells and activate Natural Killer (NK). However, HPV produces E6 and E7 proteins which can inhibit transcription regulation of interferon 3 regulatory factor to activate interferon beta thereby canceling the initial response of the innate immune system to viral infection. E7 also binds to interferon regulatory factor 1 to prevent activation of interferon alpha and beta. In addition, E6 and E7 proteins also inhibit the translocation of macrophages to virus-infected points by inhibiting the regulation of Monocyte chemotactic protein-1 (MCP-1), a chemotaxis compound.

Normally this compound will be released by virusinfected keratin cells so that macrophages will migrate to infected cells. Then, the macrophages will be activated when they bind to viral components, such as the genetic material of the virus and its capsid. Activated macrophages release inflammatory cytokines, chemokines or interferons. Compounds released by macrophages will trigger TNF-a and antibodies to kill HPV.

It is difficult to establish the role of other early HPV proteins (such as E1, E2 and E4) in the malignancy conversion process. Two structural proteins L1 and L2 are not expressed in precancerous and malignant cells, but both are important for vaccine development. 24

In cell cultures that have been transfected with E6 DNA, the CDKN2A gene encoding INK4A (p16) is usually deactivated by methylation, mutation, or deletion. INK4A inactivates the cyclin D1-CDK4 or cyclin D1-CDK6 complexes, which prevent cyclin E expression and pass through the cell cycle. Consistent disruption of the function of the CDKN2A gene in immortal cells. E6 shows that INK4A can functionally interfere with the transformation activity of E6.

Changes caused by the coding of the HPV virus cause a lesion called a cervical intrapepithelial neoplasm (CIN). CIN is divided into 3, namely CIN I which invades $1 / 3$ of the epithelium and lasts up to 3 years, changes occur on a small scale and the immune response can eliminate this stage of infection. If it continues, CIN I will change to CIN II which affects 2/3 to all parts of the epithelium. If the changes occur persistently, it will enter the CIN III stage which lasts up to 3-6 years. If the CIN progresses to CIN III and persists for 5-10 years, the abnormal cells then become invasive. This high level of CIN causes HPV DNA to be perfectly integrated into the host genome and causes disruption to the deletion of the gene coding for E2 so that the expression of E6 and E7 proteins increases drastically.

Viral and non-viral risk factors affecting the development of infected cells are listed on the left. Hormonal factors (estrogens and their derivatives) activate the human papillomavirus (HPV) promoter and facilitate the immortality of HPV-infected cells. The mutagenic agent strengthens the existing HPV DNA. They enhance progression by modifying the cellular signaling cascade that controls HPV persistence, or by causing increased expression of viral oncogene with gene-dose effects. The increased expression levels of the oncogene E6 and E7, in turn, result in increased genomic instability, which further facilitates the progression of infected cells towards invasive growth. 25

The development of human papillomavirus (HPV) infected cells into low-grade squamous intraepithelial lesions (LSIL) and high-grade squamous intraepithelial lesions (HSIL), carcinoma in situ and invasive cancer is determined by failed control mechanisms. These include intracellular control as possible by cyclindependent kinase inhibitors, paracrine signaling cascades and decreased immunological control. Paracrine control is induced by macrophages and cytokines, such as tumor necrosis factor-a, and is based on loss of interferon- $\beta$ synthesis.

Another pathway that involves blocking HPV DNA transcription and known as the concept of cellular interference factor (CIF) has been analyzed in more detail. It is triggered by paracrine stimulation of cervical epithelial cells by macrophages and tumor necrosis factor-a (TNF-a), and causes several effects on HPVimmortalized cells. These include modification of the transcription factor AP1, which is important for HPV 
gene expression, and the induction of endogenous synthesis of the antiviral interferon- $\beta$. It has been suggested that changes in the composition of AP1 mediate suppression of high-risk HPV transcription.
This pathway does not function in cervical carcinoma cells, suggesting that the TNF-a-mediated signaling cascade is interrupted during malignant transformation.

Figure 1. Classification of HPV based on the level of malignancy

Low-Risk HPV
$6.1140(42)$
43.44 5s

6.114042

(43) $44 \sqrt{5}$
Potential

high-Risk HPV

263356167

$687073] 82$
High-Risk HPV

(16) 18 31 32 33 35 (39)
45151152156158159

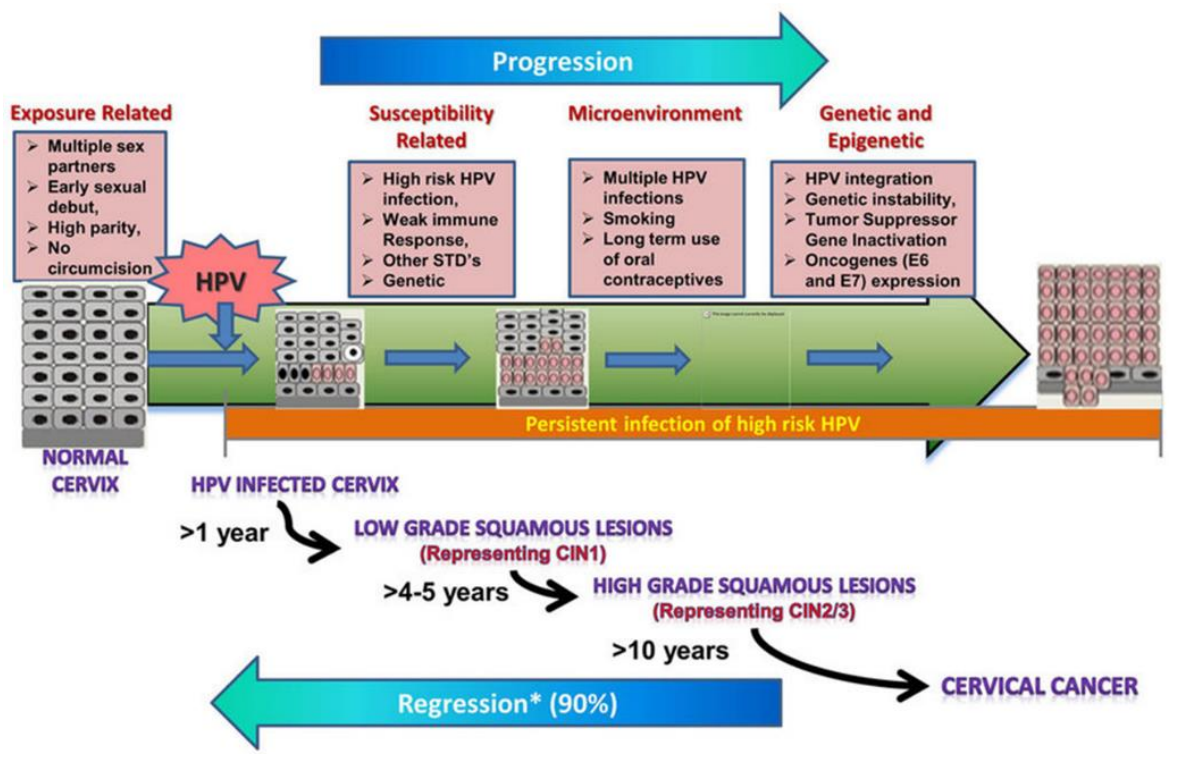

Figure 2. Natural course of HPV infection and risk factors and possible regressions

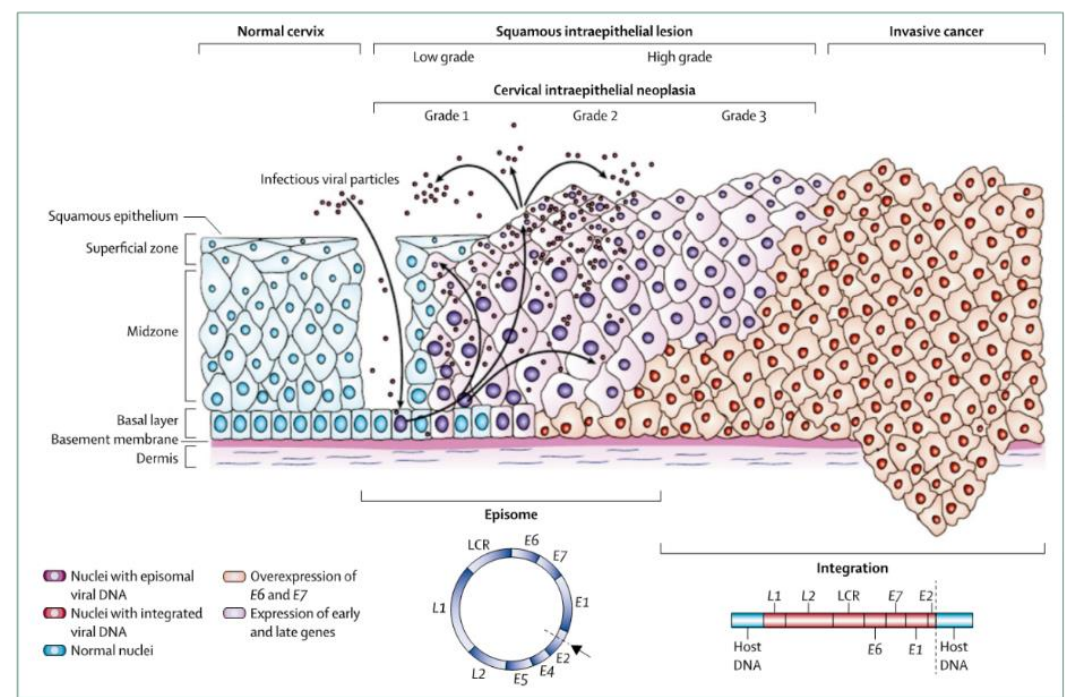

Figure 3. HPV life cycle and genome organization ${ }^{42}$ 


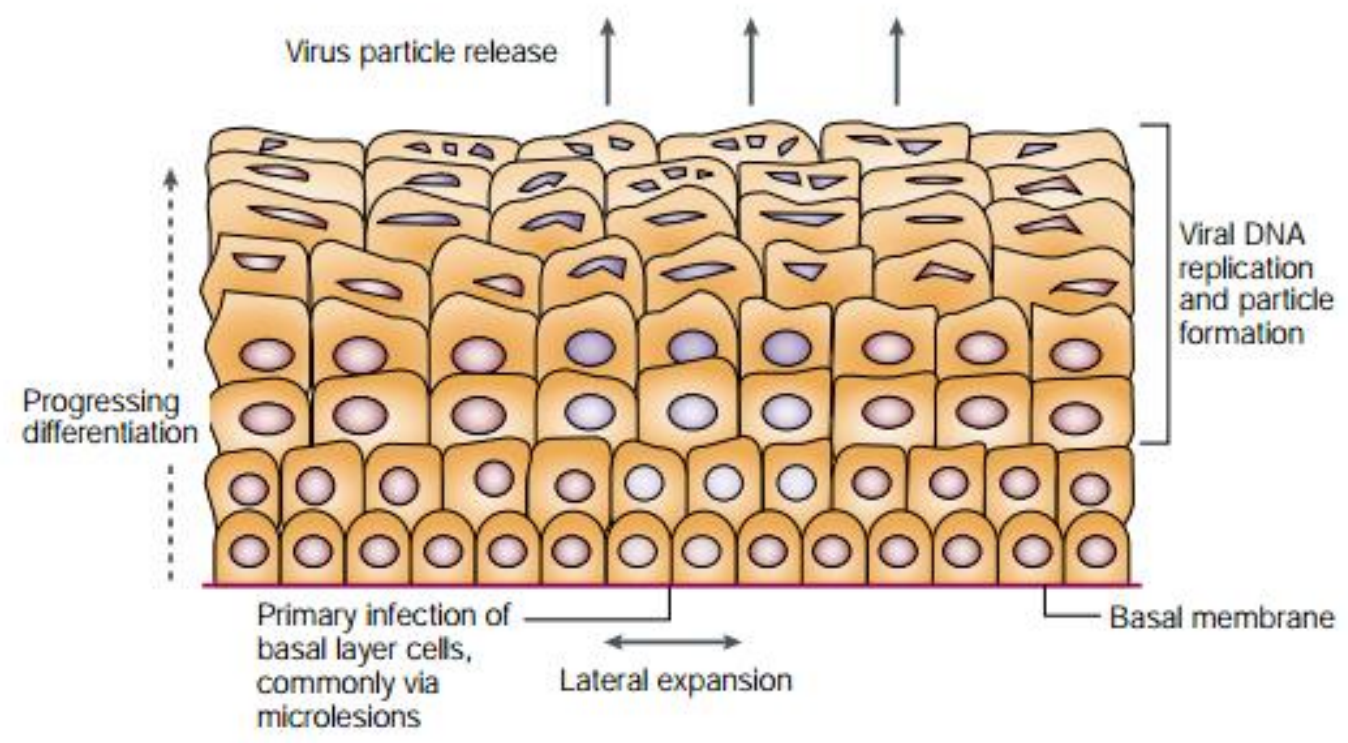

Figure 4. HPV life cycle. E5 causes lateral expansion of the cell.

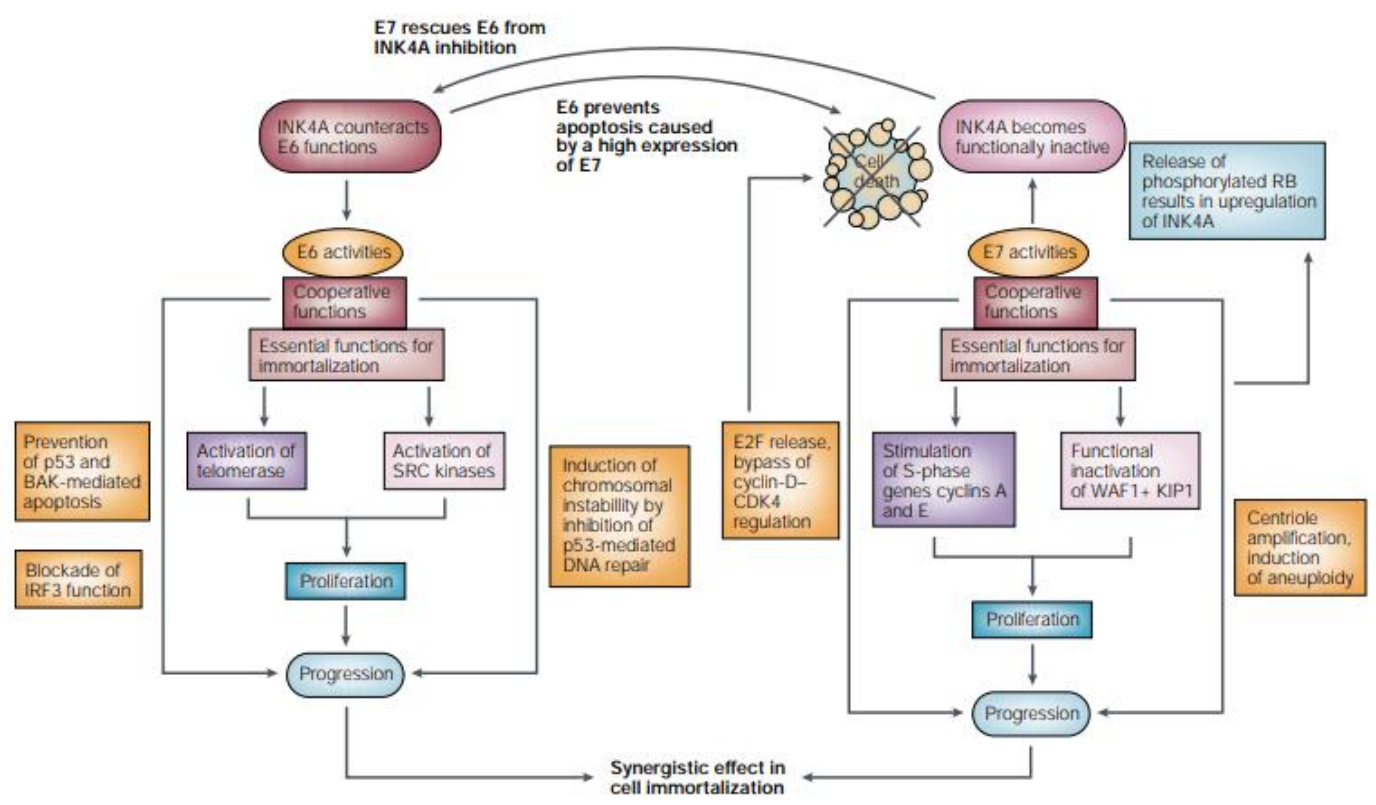

Figure 5. Functions of oncoproteins E6 and E7 and their interactions with each other. E6 and E7 work synergistically to cause excessive proliferation and prevent apoptosis induced by p53, INK4A, BAK, WAF1 + KIPI1 causing chromosomal instability. 


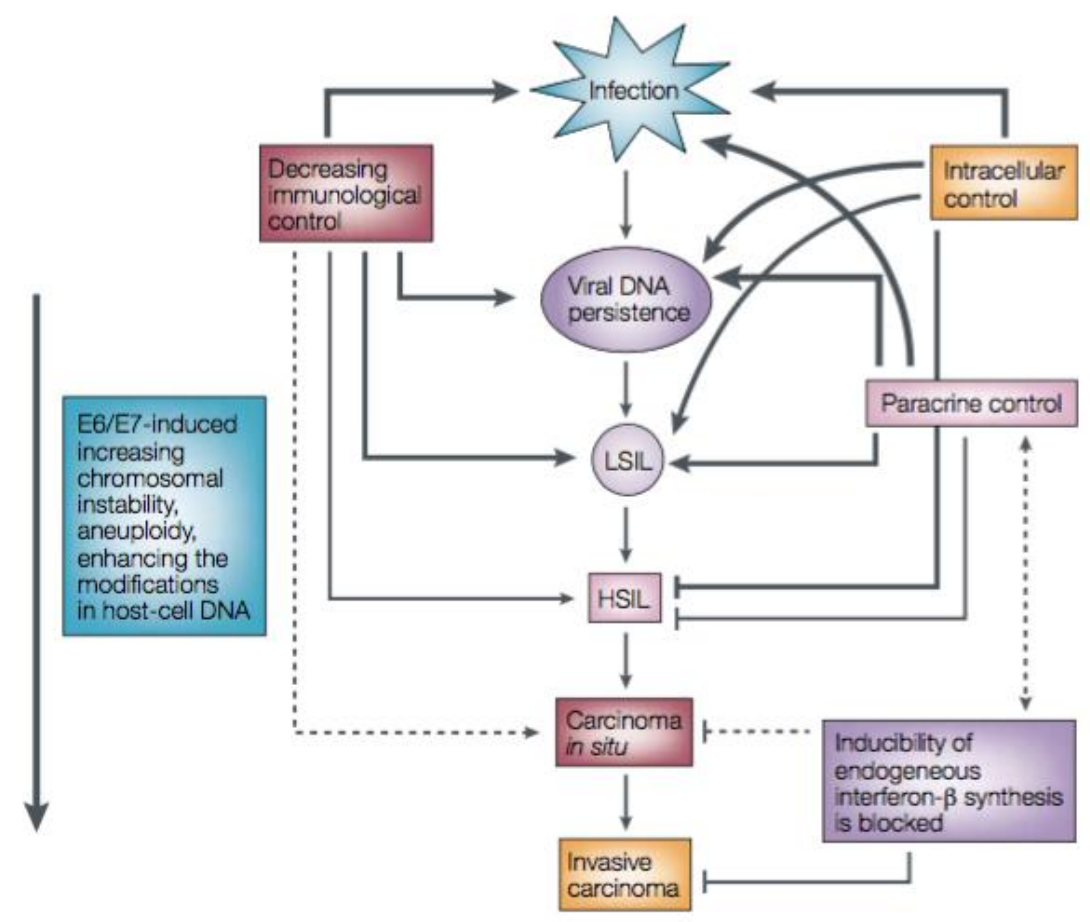

Figure 6. Host control of HPV induction.

\section{References}

1. Min KJ, Lee JK, So KA, Kim MK. Association Between Passive Smoking and the Risk of Cervical Intraepithelial Neoplasia 1 in Korean Women. $J$ Epidemiol. 2018;28(1):48-53. doi:10.2188/jea.JE20160118

2. WHO. WHO Global world health assembly adopts global strategy to accelerate cervical cancer elimination. Published 2020. Accessed October 29 , 2020. https://www.who.int/news/item/19-082020-world-health-assembly-adopts-globalstrategy-to-accelerate-cervical-cancerelimination

3. Aoki E, Yin R, Li K, et al. National screening programs for cervical cancer in Asian countries. J Gynecol Oncol. 2020;31(3). doi.org/10.3802/jgo.2020.31.e55

4. Schiffman M, Doorbar J, Wentzensen N, Monk BJ, Stanley MA, Franceschi S. Carcinogenic human papillomavirus infection. Nat Rev Dis Prim. 2016;2:1-20. doi:10.1038/nrdp.2016.86

5. Mustafa RA, Santesso N, Khatib R, et al. Systematic reviews and meta-analyses of the accuracy of HPV tests, visual inspection with acetic acid, cytology, and colposcopy. Int $J$ Gynecol Obstet. Published online 2015. doi:10.1016/j.ijgo.2015.07.024

6. Asiaf A, Ahmad ST, Mohammad SO, Zargar MA. Review of the current knowledge on the epidemiology, pathogenesis, and prevention of human papillomavirus infection. Eur J Cancer Prev. 2014;23(3):206-224. doi:10.1097/CEJ.0b013e328364f273

7. Arkhangelskaya TA, Prokhorov M V., Mazirov MA. Annual temperature dynamics of arablesoils of Vladimir opolye paleocryogenic complexes. Earth's Cryosph. 2008;12(3):80-86.

8. Development C. Key Molecular Events in Cervical Cancer Development. Published online 2019.

9. Ibeanu OA. Molecular pathogenesis of cervical cancer. Cancer Biol Ther. 2011;11(3):295-306. doi:10.4161/cbt.11.3.14686

10. Murdiyarso LS, Kartawinata M, Jenie I, Widjajahakim G, Indonesia GÁ, Hpv ÁHHPVÁL. Single and multiple high-risk and low-risk Human Papillomavirus association with 
cervical lesions of 11,224 women in Jakarta. Cancer Causes Control. Published online 2016. doi:10.1007/s 10552-016-0816-4

11. Stark H, Živković A. HPV Vaccination : Prevention of Cervical Cancer in Serbia and in Europe. Acta Fac Medicae Naissensis. 2018;371(4):5-16. doi:10.2478/afmnai-20180001

12. Human P, Hpv P, Evriarti PR, Yasmon A. Metode yang disusun dengan pendekatan telusur menimbulkan kanker pada manusia . Papillomavirus bersama Poliomavirus masuk dalam family yang sama ). Famili Namun yang paling sering ditemukan pada pasien adaah genus Alphapapillomavirus.

13. Moscicki AB, Schiffman M, Burchell A, Albero G, A. R. Giuliano, M. T. Goodman SKK and JP. Updating the Natural History of Human Papillomavirus and Anogenital Cancers. Vaccine. 2013;30(Suppl 5):F24-F33. doi:10.1016/j.vaccine.2012.05.089.Updating

14. Bosch FX, Broker TR, Forman D, et al. Comprehensive Control of Human Papillomavirus Infections and Related Diseases ¿. Vaccine. 2013;31:H1-H31. doi:10.1016/j.vaccine.2013.10.003

15. Bharti AC, Singh T, Bhat A, Pande D, Jadli M. Therapeutic startegies for human papillomavirus infection and associated cancers. Front Biosci Elit. 2018;10(4):15-73.

16. Cobo F. Human Papillomavirus Infections: From the Laboratory to Clinical Practice. 1st ed. (Publishing W, ed.). Elsevier Inc.; 2012.

17. Doorslaer K Van, Chen Z, Bernard H, et al. ICTV ICTV Virus Taxonomy Profile: Papillomaviridae. Published online 2018:989990. doi:10.1099/jgv.0.001105

18. Crosbie EJ, Einstein MH, Franceschi S, Kitchener HC. Human papillomavirus and cervical cancer. Lancet. 2013;382(9895):889899. doi:10.1016/S0140-6736(13)60022-7

19. Longworth MS, Laimins LA. Pathogenesis of Human Papillomaviruses in Differentiating
Epithelia. 2004;68(2):362-372. doi:10.1128/MMBR.68.2.362

20. Role O, Techniques D. The Human Papillomavirus ( HPV ) in Human Pathology: Description, The Human Papillomavirus ( HPV ) in Human Pathology: Description, Pathogenesis , Oncogenic Role , Epidemiology and Detection Techniques. Open Dermatol $J$. 2009;3:90-102.

doi:10.2174/1874372200903010090

21. Muñoz N, Bosch FX, De Sanjosé S, et al. Epidemiologic classification of human papillomavirus types associated with cervical cancer. $N$ Engl J Med. 2003;348(6):518-527. doi:10.1056/NEJMoa021641

22. IARC IAFROC. Monographs on the Evaluation of Carcinogenic Risks to Humans Human Papillomaviruses.; 2007.

https://monographs.iarc.fr/wpcontent/uploads/2018/06/mono90.pdf

23. Hwang E, Nottoli T, DiMaio D. HPV 16 E5 protein: expression, detection and stable complex formation with transmembrane protein in COS cells. Virology. 1995;211:227233.

24. Zhang B, Spandau DF, Roman A. E5 Protein of Human Papillomavirus Type 16 Protects Human Foreskin Keratinocytes from UV BIrradiation-Induced Apoptosis. 2002;76(1):220-231. doi:10.1128/JVI.76.1.220 